\title{
SPECKLE OBSERVATIONS OF SOLAR GRANULATION
}

\author{
C.R. DE BOER AND F. KNEER \\ Universitäts-Sternwarte, Geismarlandstr. 11 \\ D-3400 Göttingen, Federal Republic of Germany
}

Image reconstruction by means of speckle interferometry was successfully used to restore the intensity distribution of solar features and to investigate the morphology and dynamics of small-scale structures in active regions of the Sun. The observations were obtained with the Vacuum Tower Telescope $(D=70 \mathrm{~cm}, \mathrm{f}=$ $46 \mathrm{~m}$ ) at Observatorio del Teide, Tenerife, on May 17 and 20, 1991, from a plage region close to a sunspot near disc centre. Sequences of bursts consisting of 100 exposures were recorded with a broad-band filter centred at $550 \mathrm{~nm}$ (FWHM $\approx 10$ $\mathrm{nm}$, diffraction limit 0.2 arcsec). The pickup unit was a video CCD - system with an exposure time of $4 \mathrm{~ms}$ and a frame rate of three pictures per second. A description of the observing procedure and of the data handling can be found in de Boer et al. (1992). To obtain the complex Fourier phases speckle masking (Lohmann et al. 1983) was used. The speckle transfer function of the atmosphere was calculated indirectly using Korff's equation (1973). The Fried parameter $r_{0}$ was estimated with the spectral ratio technique (von der Lühe 1984). This parameter was sometimes as large as $14 \mathrm{~cm}$. With this the theoretical speckle transfer function could be determined for calculating the corrected Fourier amplitudes of the reconstruction. A new low pass filter, based on the reliability of each individual value in the Fourier plane, was applied to the amplitudes to suppress noise at high wavenumbers.

Figure 1 shows one restored image out of a time series. Small-scale structures with sizes close to the telescopic diffraction limit are discernible. The most interesting details in this image are the intensity distributions of the granules. The brightest areas sometimes occur as short bright lanes along the borders to the intergranulum with horizontal extent near the diffraction limit. Especially large granules with high horizontal velocities show such bright borders. To prove that the brightenings are real several tests were carried out (de Boer et al. 1992). There are two different explanations of this behaviour: i) Simulations of solar granulation by Steffen (1993) show enhanced upward flow in the subphotospheric layers below granular borders, because the photospheric pressure is lowest there. This could yield an enhanced energy flux and therefore enhanced intensity. ii) Computer simulations of granulation by Cattaneo at al. (1991) predict fast horizontal outflows with shocks. At the same location one can see the bright lanes. In this case the brightenings are caused by an increase of entropy in post-shock regions (see also Nesis et al. 1992).

Figure 2 gives three consecutive images of another time sequence after speckle restoration. The effective temporal distance is $90 \mathrm{sec}$. The best single exposures of the bursts show diffuse, "abnormal" granulation in the calcium plage region (de Boer \& Kneer 1992). They actually consist of many small, irregularly shaped components close to the diffraction limit. The typical granular pattern is barely visible. One notices nests and chains of bright "points" embedded in intergranular spaces. One can suppose that these chains with distances around 0.2 to 0.4 arcsec are resolved photospheric filigree "crinkles" (Dunn \& Zirker 1973). 


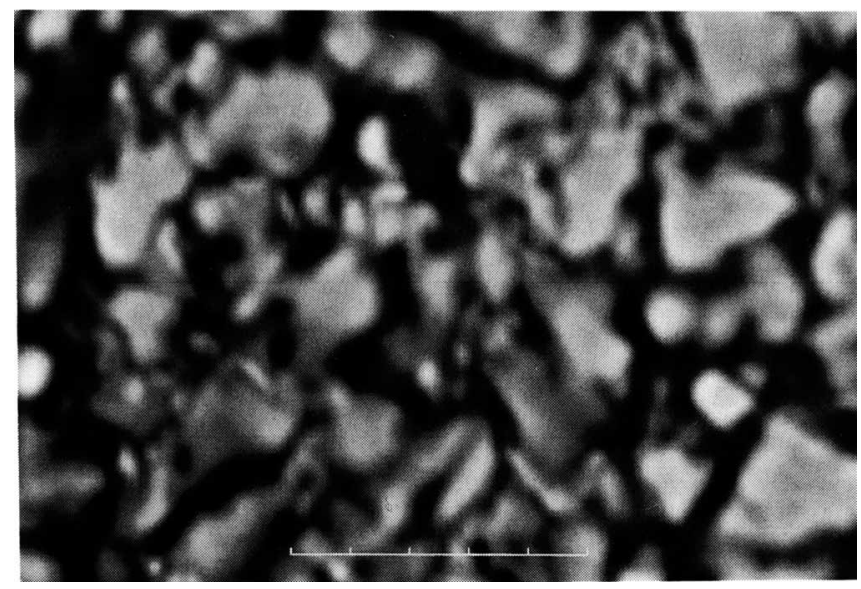

Fig. 1. Restored image of a time sequence. Distance of tick-marks: 1 arcsec

It is difficult to follow all structures from one reconstruction to the next. When it is possible one notices horizontal velocities of the "points" up to $2-3 \mathrm{~km} / \mathrm{sec}$. Obviously, considering such small spatial scales, temporal evolution also occurs on time scales shorter than $90 \mathrm{sec}$. We suppose that the bright "points" may be identified with the footpoints of small-scale magnetic fluxtubes. Their non-stationary, apparently chaotic behaviour on time scales of 1 - 2 min has consequences for the dynamical processes of interaction of magnetic fields and the gas in the solar atmosphere. The behaviour of the bright points seems to be dominant in abnormal granulation. A more detailed analysis can be found in de Boer \& Kneer (1992).

\section{Acknowledgements}

This project was supported by the Deutsche Forschungsgemeinschaft through grants Kn 152/5-1 and Kn 152/5-2. The Vacuum Tower Telescope at the Spanish Observatorio del Teide of the Instituto de Astrofísica de Canarias is operated by the Kiepenheuer - Institut für Sonnenphysik in Freiburg.

\section{References}

Cattaneo, F., Brummel, N.H., Toomre, J., Malagoli, A., Hurlburt, N. E.: 1991, Astrophys. J. 370, 282

de Boer, C. R., Kneer, F., Nesis, A.: 1992, Astron. Astrophys. 257, L3

de Boer, C. R., Kneer, F.: 1992, Astron. Astrophys. 264, L24

Dunn, R. B., Zirker, J. B.: 1973, Solar Phys. 33, 281

Korff, D.: 1973, J. Opt. Soc. Am. 63, 971

Lohmann, A. W., Weigelt, G., Wirnitzer, B.: 1983, Appl. Opt. 22, 337

Nesis, A., Bogdan, T. J., Cattaneo, F., Hanslmeier, A., Knölker, M., Malagoli, A.: 1992, Astrophys. J. 399, L99

Pehlemann, E., von der Lühe, O.: 1989, Astron. Astrophys. 216, 337

Steffen, M.: 1993, in preparation,

von der Lühe, O.: 1984, J. Opt. Soc. Am. A1, 510 

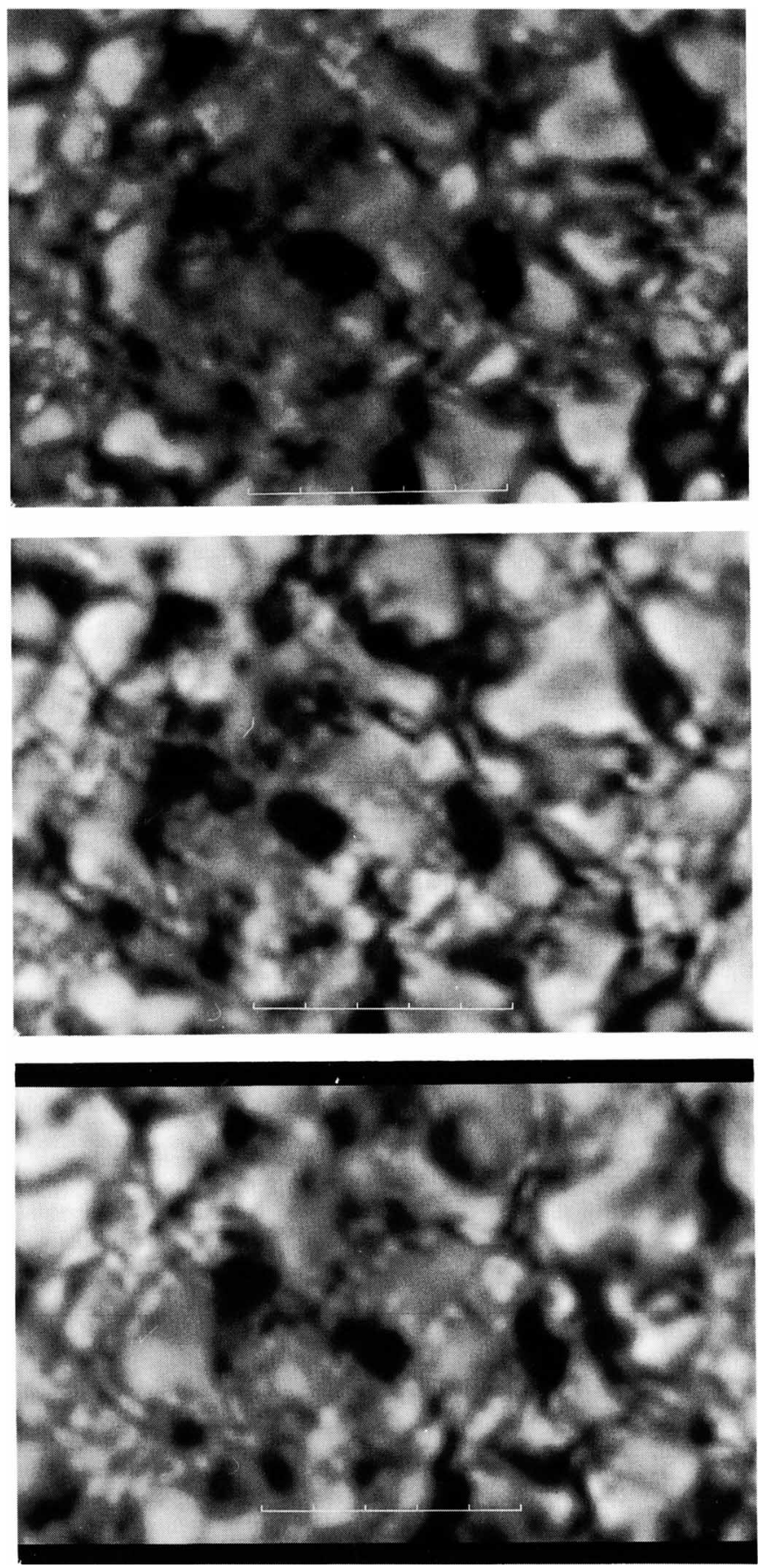

Fig. 2. Three consecutive restored images from a time sequence. Distance of tick-marks: 1 arcsec 